\title{
New Results on the Existence of Periodic Solutions for Rayleigh Equation with State-Dependent Delay
}

\author{
Cemil Tunç \\ Department of Mathematics, Faculty of Sciences, \\ YüzüncüYıl University, 65080, Van, Turkey \\ E-mail: cemtunc@yahoo.com
}

\begin{abstract}
We give certain sufficient conditions for the existence of periodic solutions to a Rayleigh-type equation with state-dependent delay. With this work we extend and improve some known results in the literature.
\end{abstract}

Keywords: non-linear; periodic solution; Rayleigh equation; second order; statedependent delay.

\section{$1 \quad$ Introduction}

It is well known that the British mathematical physicist Lord Rayleigh [1], the Nobel Prize laureate for Physics in 1904, introduced an equation of the form

$$
x^{\prime \prime}(t)+f\left(x^{\prime}(t)\right)+a x(t)=0
$$

to model the oscillations of a clarinet reed; for details, see Wang and Zhang [2].This equation was named after Lord Rayleigh, who studied equations of this type in relation to problems in acoustics.

In 1977 and 1985 respectively, Gains and Mawhin [3] and Deimling [4] introduced some continuation theorems and applied them to discuss the existence of periodic solutions to ordinary differential equations. In particular, a specific example was given in [3, p. 99] of how $T$-periodic solutions can be obtained using the established theorems for the differential equation of the form

$$
x^{\prime \prime}(t)+f\left(x^{\prime}(t)\right)+g(t, x(t))=0 .
$$

In this direction, in recent years, Alzabut and Tunç [5], Chen [6], Chen, et al. [7], Liu [8], Lu and Ge [9], Lu, et al. ([10], [11]), Tunç and Sirma [12], Zhou and Tang [13],Wang and Cheng [14],Wang and Yan [15,16], Wang and Zhang [2], etc., continued to discuss the existence of periodic solutions to Rayleigh equations and modified Rayleigh equations with and without delay. These researchers obtained many new results concerning the existence of periodic solutions to the mentioned equations. It should be noted that throughout these papers, the mentioned authors took into consideration the results from [3] to prove their main results.

Received February $28^{\text {th }}, 2013$, Revised June $8^{\text {th }}, 2013$, Accepted for publication June $17^{\text {th }}, 2013$.

Copyright $\odot 2013$ Published by ITB Journal Publisher, ISSN: 2337-5760, DOI: 10.5614/j.math.fund.sci.2013.45.2.5 
In this paper, we consider a Rayleigh-type equation with state-dependent delay of the form

$$
x^{\prime \prime}(t)+\psi(x(t)) x^{\prime}(t)+f\left(t, x^{\prime}(t-\sigma)\right)+g(t, x(t-\tau(t, x(t))))=p(t)
$$

and its auxiliary equation

$$
x^{\prime \prime}(t)+\lambda \psi(x(t)) x^{\prime}(t)+\lambda f\left(t, x^{\prime}(t-\sigma)\right)+\lambda g(t, x(t-\tau(t, x(t))))=\lambda p(t),
$$

where $\lambda \in(0,1), \sigma \geq 0, f, g \in C\left(\Re^{2}, \Re\right), \tau \in C\left(\mathfrak{R}^{2}, \Re^{+}\right), \mathfrak{R}^{+}=[0, \infty), f$ and $g$ are $2 \pi$ - periodic in $t, f(t, 0)=g(t, 0)=0$ for $t \in \mathfrak{R}, \psi, p \in C(\mathfrak{R}, \mathfrak{R}), \tau$ and $p$ are $2 \pi$-periodic in $t$, and $\int_{0}^{2 \pi} p(t) d t=0$.

We have established two new theorems on the existence of periodic solutions of Eq. (1). This paper was inspired by the results established in the aforementioned papers and in the literature. Our aim was to generalize and improve the results of Zhou and Tang [13] and Wang and Yan [15]. This paper is also a contribution to the subject in the literature and it may be useful for researchers who work on the qualitative behaviors of solutions.

It should also be noted that, in 2012, Alzabut and Tunç [5] discussed the existence of periodic solutions for a type of Rayleigh equation with statedependent delay of the form

$$
x^{\prime \prime}(t)+f\left(t, x^{\prime}(t)\right)+g(t, x(t-\tau(t, x(t))))=p(t) .
$$

The authors of [5] utilized the continuation theorem of degree theory to obtain sufficient conditions for the existence of periodic solutions of the above equation. The main result in [5] has been proved by bypassing the boundedness of $g$ and the integral condition on $p$. When we compare Eq. (2) with the equation discussed in [5], it can been seen that Eq. (2) is different from that studied in [5]. Moreover, the authors of [5] utilized the continuation theorem of degree theory to obtain sufficient conditions for the existence of periodic solutions of the above equation; however, here we did not utilize the continuation theorem of degree theory. The conditions to be established and the method to be utilized in this study were different from those used in [5]. The cases show the difference between our results and those from [5].

For the sake of convenience, let

$$
C_{2 \pi}=\{x: x \in C(\Re, \Re), x(t+2 \pi)=x(t), \forall t \in \mathfrak{R}\},
$$




$$
\begin{aligned}
& \|x\|_{0}=\max _{t \in[0,2 \pi]}|x(t)|, \\
& \left\|x^{\prime}\right\|_{0}=\max _{t \in[0,2 \pi]} \mid x^{\prime}(t) .
\end{aligned}
$$

\section{Statement of Main Results}

The following lemma plays a key role in proving the main results.

Lemma. Let $x(t)$ be a continuous differentiable $T$-periodic function $(T>0)$.

Then for any $t_{*} \in(-\infty, \infty)$

$$
\max _{t \in\left[t_{*}, t_{*}+T\right]}|x(t)| \leq\left|x\left(t_{*}\right)\right|+\frac{1}{2} \int_{0}^{T}\left|x^{\prime}(s)\right| d s .
$$

Proof. Let $t^{*} \in\left[t_{*}, t_{*}+T\right]$ such that $x\left(t^{*}\right)=\max _{t \in\left[t_{*}, t_{*}+T\right]}|x(t)|$. Then

$$
\left|x\left(t^{*}\right)\right|=\left|x\left(t_{*}\right)+\int_{t_{*}}^{t^{*}} x^{\prime}(s) d s\right| \leq\left|x\left(t_{*}\right)\right|+\int_{t_{*}}^{t^{*}}\left|x^{\prime}(s)\right| d s
$$

and

$$
\left|x\left(t^{*}\right)\right|=\left|x\left(t^{*}-T\right)\right|=\left|x\left(t_{*}\right)-\int_{t^{*}-T}^{t_{*}} x^{\prime}(s) d s\right| \leq\left|x\left(t_{*}\right)\right|+\int_{t^{*}-T}^{t_{*}}\left|x^{\prime}(s)\right| d s .
$$

Combining the last two estimates, we obtain

$$
\left|x\left(t^{*}\right)\right| \leq\left|x\left(t_{*}\right)\right|+\frac{1}{2} \int_{t^{*}-T}^{t^{*}}\left|x^{\prime}(s)\right| d s=\left|x\left(t_{*}\right)\right|+\frac{1}{2} \int_{0}^{T}\left|x^{\prime}(s)\right| d s .
$$

The proof is complete.

Our first main result is the following theorem:

Theorem 1. We assume that there exist constants $a_{1}>0, r_{1}, r_{2} \geq 0, d>0, K>0$ and $M>0$ such that the following conditions hold:

(H1) $|\psi(x)| \leq a_{1}$ for all $x \in \mathfrak{R}$,

(H2) $|f(t, x)| \leq r_{1}|x|+K$ for all $(t, x) \in \mathfrak{R}^{2}$,

(H3) $x g(t, x(t-\tau(t, x)))>0$ and $|g(t, x(t-\tau(t, x)))|>r_{1}|x|+K$ for all $t \in \mathfrak{R},|x|>d$, 
(H4) $g(t, x(t-\tau(t, x)))>r_{2} x-M$ for all $t \in \Re, x \leq-d$.

If

$$
2 \pi\left\{\left(a_{1}+r_{1}\right)+(\pi+1) r_{2}\right\}<1,
$$

then Eq. (1) has at least one $2 \pi$-periodic solution.

Proof. We reconsider the auxiliary equation, Eq. (2). Let $x(t)$ be any $2 \pi-$ periodic solution of Eq. (2). Then, integrating both sides of Eq. (2) from 0 to $2 \pi$, it follows that

$$
\begin{gathered}
\left.\int_{0}^{2 \pi} \psi(x(s)) x^{\prime}(s)\right) d s+\int_{0}^{2 \pi}\left\{f\left(s, x^{\prime}(s-\sigma)\right)+g(s, x(s-\tau(s, x(s))))\right\} d s=0 \\
\Rightarrow \int_{x(0)}^{x(2 \pi)} \psi(u) d u+\int_{0}^{2 \pi}\left\{f\left(s, x^{\prime}(s-\sigma)\right)+g(s, x(s-\tau(s, x(s))))\right\} d s=0 \\
\Rightarrow \int_{0}^{2 \pi}\left\{f\left(s, x^{\prime}(s-\sigma)\right)+g(s, x(s-\tau(s, x(s))))\right\} d s=0 .
\end{gathered}
$$

Hence, it follows that there exists a $t_{1} \in[0,2 \pi]$ such that

$$
f\left(t_{1}, x^{\prime}\left(t_{1}-\sigma\right)\right)+g\left(t_{1}, x\left(t_{1}-\tau\left(t_{1}, x\left(t_{1}\right)\right)\right)\right)=0 .
$$

We claim that there exists a $t^{*} \in[0,2 \pi]$ such that

$$
\left|x\left(t^{*}\right)\right| \leq\left\|x^{\prime}\right\|_{0}+d
$$

Case 1. Let $r_{1}=0$. Then

$$
|f(t, x)| \leq r_{1}|x|+K \Rightarrow|f(t, x)| \leq K .
$$

From the last estimate and (5), we have

$$
\left|g\left(t_{1}, x\left(t_{1}-\tau\left(t_{1}, x\left(t_{1}\right)\right)\right)\right)\right| \leq K .
$$

The last estimate together with the assumption

$$
|g(t, x(t-\tau(t, x)))|>r_{1}|x|+K,|x|>d,
$$

implies that 


$$
\left|x\left(t_{1}-\tau\left(t_{1}, x\left(t_{1}\right)\right)\right)\right| \leq d .
$$

Case 2. Let $r_{1}>0$. If $\left|x\left(t_{1}-\tau\left(t_{1}, x\left(t_{1}\right)\right)\right)\right|>d$, then it follows from the estimate (5) and the assumptions of (H2) and (H3) that

$$
r_{1}\left|x\left(t_{1}-\tau\left(t_{1}, x\left(t_{1}\right)\right)\right)\right|+K<\left|g\left(t_{1}, x\left(t_{1}-\tau_{1}\left(t_{1}, x\right)\right)\right)\right| \leq r_{1}\left|x^{\prime}\left(t_{1}-\sigma\right)\right|+K .
$$

From the first and last terms of the last estimate it follows that

$$
\left|x\left(t_{1}-\tau\left(t_{1}, x\left(t_{1}\right)\right)\right)\right| \leq\left|x^{\prime}\left(t_{1}-\sigma\right)\right| \leq\left\|x^{\prime}\right\|_{0} \leq\left\|x^{\prime}\right\|_{0}+d .
$$

We note that $x(t)$ is periodic and there exists a $t^{*} \in[0,2 \pi]$ such that

$$
\left|x\left(t^{*}\right)\right| \leq\left\|x^{\prime}\right\|_{0}+d
$$

holds.

From Lemma, for all $t^{*} \in(-\infty, \infty)$, we have

$$
\begin{aligned}
\|x\|_{0} & \leq\left|x\left(t^{*}\right)\right|+\frac{1}{2} \int_{0}^{2 \pi}\left|x^{\prime}(s)\right| d s \\
& \leq(\pi+1)\left\|x^{\prime}\right\|_{0}+d \\
\Rightarrow\|x\|_{0} & \leq(\pi+1)\left\|x^{\prime}\right\|_{0}+d .
\end{aligned}
$$

Hence, for all $t_{*} \in(-\infty, \infty)$, we can write

$$
\left\|x^{\prime}\right\|_{0} \leq\left|x^{\prime}\left(t_{*}\right)\right|+\frac{1}{2} \int_{0}^{T}\left|x^{\prime \prime}(s)\right| d s
$$

Since $x(0)=x(2 \pi)$, then it follows from Rolle's Theorem that there exists a $\exists c \in(0,2 \pi)$ such that $x^{\prime}(c)=0$. Let $t_{*}=c$. Hence, we get

$$
\left\|x^{\prime}\right\|_{0} \leq \frac{1}{2} \int_{0}^{2 \pi}\left|x^{\prime \prime}(s)\right| d s
$$

Let

$$
\begin{aligned}
& E_{1}=\{t: t \in[0,2 \pi], x(t-\tau(t, x(t)))>d\}, \\
& E_{2}=\{t: t \in[0,2 \pi], x(t-\tau(t, x(t)))<-d\}
\end{aligned}
$$

and 


$$
E_{3}=\{t: t \in[0,2 \pi],|x(t-\tau(t, x(t)))| \leq d\} .
$$

From (4) it follows that

$$
\int_{E_{1}}|g(s, x(s-\tau(s, x(s))))| d s \leq \int_{0}^{2 \pi} \mid f\left(s, x^{\prime}(s-\sigma)\left|d s+\left(\int_{E_{2}}+\int_{E_{3}}\right)\right| g(s, x(s-\tau(s, x(s)))) \mid d s .\right.
$$

Hence, we can get

$$
\begin{gathered}
\left\|x^{\prime}\right\|_{0} \leq \frac{1}{2} \int_{0}^{2 \pi}\left|x^{\prime \prime}(s)\right| d s \quad \leq \frac{1}{2} \int_{0}^{2 \pi}\left|f\left(s, x^{\prime}(s-\sigma)\right)\right| d s+\frac{1}{2} \int_{0}^{2 \pi}\left|\psi(x(s)) x^{\prime}(s)\right| d s \\
+\frac{1}{2} \int_{0}^{2 \pi}|g(s, x(s-\tau(s, x(s))))| d s+\frac{1}{2} \int_{0}^{2 \pi}|p(s)| d s \leq \frac{1}{2} \int_{0}^{2 \pi}\left|f\left(s, x^{\prime}(s-\sigma)\right)\right| d s \\
+\frac{1}{2} \int_{0}^{2 \pi}|\psi(x(s))|\left|x^{\prime}(s)\right| d s \quad+\frac{1}{2}\left(\int_{E_{1}}+\int_{E_{2}}+\int_{E_{3}}\right)|g(s, x(s-\tau(s, x(s))))| d s+\pi\left\|_{0}\right\|_{0} \\
\leq \int_{0}^{2 \pi}\left|f\left(s, x^{\prime}(s-\sigma)\right)\right| d s+a_{1} \pi\left\|x^{\prime}\right\|_{0} \\
+\left(\int_{E_{2}}+\int_{E_{3}}\right)|g(s, x(s-\tau(s, x(s))))| d s+\pi\|p\|_{0} \\
\leq 2 \pi\left\{\left(a_{1}+r_{1}\right)\left\|x^{\prime}\right\|_{0}+r_{2}\|x\|_{0}\right\}+2 \pi\left(K+M+g_{d}\right)+\pi\|p\|_{0} \\
\left.\leq 2 \pi\left\{\left(a_{1}+r_{1}\right)+(\pi+1) r_{2}\right\}\left\|x^{\prime}\right\|_{0}\right\} \\
+2 \pi\left(K+M+g_{d}+r_{2} d\right)+\pi\|p\|_{0} \\
\Rightarrow\left\|x^{\prime}\right\|_{0} \leq 2 \pi\left\{\left(a_{1}+r_{1}\right)+(\pi+1) r_{2}\right\}\left\|x^{\prime}\right\|_{0}+2 \pi\left(K+M+g_{d}+r_{2} d\right)+\pi\left\|_{p}\right\|_{0} \\
\Leftrightarrow\left\|x^{\prime}\right\|_{0} \leq \frac{2 \pi\left(K+M+g_{d}+r_{2} d\right)+\pi\|p\|_{0}}{1-2 \pi\left\{\left(a_{1}+r_{1}\right)+(\pi+1) r_{2}\right\}}=M_{1},
\end{gathered}
$$

where

$$
g_{d}=\max _{t \in[0,2 \pi],|x| \leq d}|g(t, x)| .
$$

It also follows from the estimate

$$
\|x\|_{0} \leq(\pi+1)\left\|x^{\prime}\right\|_{0}+d
$$

that

$$
\|x\|_{0} \leq(\pi+1) M_{1}+d \text {. }
$$

This completes the proof of Theorem 1. 
Our second main result is the following theorem:

Theorem 2. We assume that there exist constants $a_{1}>0, r_{1}, r_{2} \geq 0, d>0$, $K>0$ and $M>0$ such that the following conditions hold:

(H1) $|\psi(x)| \leq a_{1}$ for all $x \in \mathfrak{R}$,

(H2) $|f(t, x)| \leq r_{1}|x|+K$ for all $(t, x) \in \mathfrak{R}^{2}$,

(H3) $\quad x g(t, x(t-\tau(t, x)))>0$ and $|g(t, x(t-\tau(t, x)))|>r_{1}|x|+K$ for all $t \in \mathfrak{R},|x|>d$,

(H4) $g(t, x(t-\tau(t, x))) \leq r_{2} x+M$ for all $t \in \mathfrak{R}, x \geq d$.

If

$$
2 \pi\left\{\left(a_{1}+r_{1}\right)+(\pi+1) r_{2}\right\}<1
$$

then Eq. (1) has at least one $2 \pi$-periodic solution.

Proof. Since the proof of Theorem 2 is similar to the proof of Theorem 1 it is omitted.

Remark 1. When $\psi(x) \equiv 0$ and $\tau(t, x(t)) \equiv \tau$ (constant) and $r_{1}=r_{2}=0$, then the conditions of Theorem 1 and Theorem 2 reduce to those of Wang and Yan [15], Theorem 2.1 and Theorem 2.2], respectively. Therefore, our results generalize and improve the corresponding results in [15].

Remark 2. When $\psi(x) \equiv 0$ and $\tau(t, x(t)) \equiv \tau(t)$, then the conditions of Theorem 1 and Theorem 2 reduce to those of Zhou and Tang [13, Theorem 2.1 and Theorem 2.2], respectively. Therefore, our results generalize and improve the results in [13].

\section{Conclusions}

A Rayleigh-type equation with state-dependent delay was considered. The existence of periodic solutions to this equation was investigated. Without using the continuation theorem of degree theory, we obtained sufficient conditions for the existence of periodic solutions. With this paper, we have extended and improved some results in the literature. 


\section{Acknowledgements}

The author would like to express his sincere appreciation to the anonymous reviewers for their helpful comments, corrections and suggestions that helped improving the presentation and quality of this work.

\section{References}

[1] Strutt, J.W. [Lord Rayleigh], Theory of Sound, 1, Dover Publications, New York, 1877, re-issued 1945.

[2] Wang, Y. \& Zhang, L., Existence of Asymptotically Stable Periodic Solutions of A Rayleigh Type Equation, Nonlinear Anal., 71(5-6), pp. 1728-1735, 2009.

[3] Gains, R.E. \& Mawhin, J.L., Coincidence Degree and Nonlinear Differential Equations, Lecture Notes in Mathematics, 568, Springer, Berlin, 1977.

[4] Deimling, K., Nonlinear Functional Analysis, Springer, Berlin, 1985.

[5] Alzabut, J. \& Tunç, C., Existence of Periodic Solutions for A Type of Rayleigh Equation with State-Dependent Delay, Electron. J. Diff. Equ., 2012(77), pp. 1-8, 2012.

[6] Chen, F.D., Existence and Uniqueness of Almost Periodic Solutions for Forced Rayleigh Equations, Ann. Differential Equations, 17(1), pp. 1-9, 2001.

[7] Chen, F.D., Chen, X.X., Lin, F.X. \& Shi, J.L., Periodic Solution and Global Attractivity of A Class of Differential Equations with Delays, Acta Math. Appl. Sinica, 28(1), pp.55-64, 2005.

[8] Liu, F., On The Existence of The Periodic Solutions of Rayleigh Equation, Acta Math. Sinica, 37(5), pp. 639-644, 1994.

[9] Lu, S.P. \& Ge, W.G., Some New Results on the Existence of Periodic Solutions to A Kind of Rayleigh Equation with A Deviating Argument, Nonlinear Anal., 56, pp. 501-514, 2004.

[10] Lu, S.P., Ge, W.G. \& Zheng, Z.X., Periodic Solutions for A Kind of Raleigh Equation with A Deviating Argument, Acta Math. Sinica, 47(2), pp. 299-304, 2004.

[11] Lu, S.P., Ge, W.G. \& Zheng, Z.X., Periodic Solutions for a Kind of Rayleigh Equation with A Deviating Argument, Appl. Math. Lett., 17, pp. 443-449, 2004.

[12] Tunç, C. \& Sirma, A., Stability Analysis of A Class of Generalized Neutral Equations, J. Comput. Anal. Appl., 12(4), pp. 754-759, 2010.

[13] Zhou, Y. \& Tang, X., On Existence of Periodic Solutions of Rayleigh Equation of Retarded Type, J. Comput. Appl. Math., 203(1), pp. 1-5, 2007. 
[14] Wang, G.Q. \& Cheng, S.S., A Priori Bounds for Periodic Solutions of A Delay Rayleigh Equation, Appl. Math. Lett., 12, pp. 41-44, 1999.

[15] Wang, G.Q. \& Yan, J.R., On Existence of Periodic Solutions of The Rayleigh Equation of Retarded Type, Internat. J. Math. Math. Sci., 23(1), pp. 65-68, 2000.

[16] Wang, G.Q. \& Yan, J.R., Existence Theorem of Periodic Positive Solutions for The Rayleigh Equation of Retarded Type, Portugal. Math., 57(2), pp. 153-160, 2000. 